Wilfried Andlauer Claudia Stumpf Konstanze Frank Peter Fürst

\section{Absorption and metabolism of anthocyanin cyanidin-3-glucoside in the isolated rat small intestine is not influenced by ethanol}

Received: 20 September 2002

Accepted: 20 February 2003
W. Andlauer · C. Stumpf · K. Frank · P. Fürst Institute for Biological Chemistry

\& Nutrition

University of Hohenheim

Stuttgart, Germany

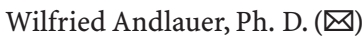

Haute Ecole Valaisanne

Route du Rawyl 47

1950 Sion 2, Switzerland

Tel.: + 41-27/606-8637

Fax: +41-27/606-8515

E-Mail: anw@hevs.ch
Summary Anthocyanins are receiving renewed attention for their positive health attributes. High intakes and an adequate absorption rate of anthocyanins are necessary for efficient protection, though other dietary agents might influence absorption efficacy. The aim of this study was to investigate intestinal handling of luminally administered cyanidin-3-glucoside in the absence and presence of ethanol in an isolated preparation of luminally and vascularly perfused rat small intestine. A synthetic perfusate free from blood components was used as vascular medium, with a perfluorocarbon as the oxygen carrier. Luminal media consisted of a bicarbonate buffered sodium chloride solution in water or in water/ethanol (95/5), spiked with cyanidin-3-glucoside. Absorption rate of cyanidin-3-glucoside was $4.3 \% \pm 3.2(\mathrm{n}=5)$. Ethanol $(5 \%)$ had no significant influence on absorption rate $(2.9 \% \pm 1.8$, $\mathrm{n}=5$ ). Irrespective of the presence of ethanol, the majority of the absorbed cyanidin-3-glucoside appeared unchanged, besides some cyanidin-3-glucoside-conjugate.

Key words cyanidin-3-glucoside - anthocyanin - intestinal metabolism - intestinal absorption - rat

\section{Introduction}

Anthocyanins are major phenolic compounds from fruits, beans, cereals, vegetables and beverages like juices and especially red wine $[1,2]$. These flavonoids are receiving renewed attention for their positive health attributes. Consumption of anthocyanins is associated with a reversal of age-dependent neuronal degeneration [3], reduced platelet aggregation [4] as well as significant vaso-protective and anti-inflammatory effects [5]. Although our diet contains relatively high amounts of these protective anthocyanins, available research on absorption and metabolic handling is scarce. Few studies have been performed with mixtures of anthocyanins from Vaccinium myrtillus extracts [6], elderberry juice [7] and red wine [8]. In all these studies, low anthocyanin absorption rates were estimated. Recently, hu- man and animal studies focused on absorption of purified cyanidin glycosides [9-11]. These authors observed intact cyanidin glycosides in plasma and tissue compartments and also estimated very low absorption rates.

However, high intakes and an adequate absorption rate of anthocyanins would be necessary for efficient protection. Many factors affect the fate of ingested compounds. Besides the dose, the matrix in which the compounds are ingested is of great importance. It might be conceivable that other dietary agents influence the efficacy of anthocyanin absorption. The presence of compounds liable to bind or solubilize anthocyanins could modify the extent of absorption and metabolism. Ethanol, in particular, might affect absorption or influence metabolism. Consequently, it has been speculated that the presence of alcohol in red wine improves flavonoid availability by increasing intestinal absorp- 
tion [12]. Yet, the impact of ethanol on intestinal absorption of anthocyanins has not been investigated.

The aim of the present study was to evaluate absorption and metabolism of cyanidin-3-glucoside in the presence and absence of ethanol by using an isolated rat small intestine (duodenum, jejunum and ileum) [13,14]. This organ perfusion model facilitates direct investigation of luminal disappearance and venous appearance of administered compounds, thereby allowing the estimation of intestinal absorption.

\section{Materials and methods}

\section{Animals}

Male Sprague-Dawley rats (CD-rats), $40 \mathrm{~d}$ old and weighing about $170 \mathrm{~g}$, were obtained from Charles River (Sulzfeld, Germany). Rats were fed a cornstarch-based cyanidin-3-glucoside-free synthetic diet (Altromin C1000 , Altromin International $\mathrm{GmbH}$, Lage, Germany) for 5 days. Animals were provided with free access to tap water and food. For perfusions with cyanidin-3-glucoside animals weighed $213.1 \pm 7.1 \mathrm{~g}(\mathrm{n}=5)$ and for perfusions with cyanidin-3-glucoside and ethanol 217.2 \pm $15.2 \mathrm{~g}(\mathrm{n}=5)$.

\section{Vascularly and luminally perfused rat small intestine}

The small intestine was prepared in rats as described elsewhere [14, 15]. Luminal media consisted of $114.6 \pm$ $23.7 \mu \mathrm{mol} / \mathrm{L}$ cyanidin-3-glucoside (amount applied $3780.2 \pm 782.3 \mathrm{nmol}, \quad \mathrm{n}=5), \quad 135 \mathrm{mmol} / \mathrm{L} \quad \mathrm{NaCl}$, $20 \mathrm{mmol} / \mathrm{L} \mathrm{NaHCO}_{3}$ and $10 \mathrm{mmol} / \mathrm{L}$ ascorbic acid at $\mathrm{pH}$ 7.2. In case of perfusions with cyanidin-3-glucoside and ethanol ( $5 \mathrm{~mL}$ per $95 \mathrm{~mL}$ luminal media), luminal media were spiked with $130.1 \pm 39.6 \mu \mathrm{mol} / \mathrm{L}$ cyanidin-3glucoside (amount applied $4294.0 \pm 1308.2 \mathrm{nmol}, \mathrm{n}=5$ ).

Vascular perfusion medium consisted of a perfluorotributylamine (ABCR, Karlsruhe, Germany) emulsion in Krebs-buffer containing $10 \mathrm{mmol} / \mathrm{L}$ glucose and additional $0.6 \mathrm{mmol} / \mathrm{L}$ glutamine, gassed with $5 \%$ carbon dioxide in oxygen ( $\mathrm{pH}$ 7.4). The perfluorotributylamine $(200 \mathrm{~g} / \mathrm{L})$ was emulsified with polyoxyproylene-polyoxyethylene copolymer ( $25.6 \mathrm{~g} / \mathrm{L}$ Pluronic, $\mathrm{F}^{-68^{\circledR}}{ }^{\circledR}, \mathrm{BASF}$, Ludwigshafen, Germany) in sterile, pyrogene-free water, using a high-pressure homogenizer (Mouton-Gaulin LAB 60/60-10TBS, APV Gaulin GmbH, Lübeck, Germany) to an average diameter of $0.2 \mu \mathrm{m}$.

Oxygen uptake and acid-base homeostasis were carefully controlled (Clark $\mathrm{pO}_{2}$-electrode and $\mathrm{pH}$-electrode integrated in an ABL 30 Acid-Base Analyzer; Radiometer, Copenhagen, Denmark). Glucose, lactate and pyruvate were determined photometrically by using enzymatic test kits (Monotest; Boehringer Mannheim,
Germany). For glucose the MPR3 Glucose/GOD-Perid ${ }^{\circledR}$ test kit (glucose oxidase, peroxidase; $\mathrm{ABTS}^{\circledR}$; Boehringer Mannheim, Germany), for lactate the MPR3 lactate test kit (lactate dehydrogenase; $\mathrm{NAD}^{+}$) and for pyruvate the MPR1 pyruvate test kit (lactate dehydrogenase; NADH) were used.

The study was approved by the Regierungspräsidium Stuttgart, Germany.

\section{Sampling and sample preparation}

Vascular $(50 \mathrm{~mL})$ and luminal $(5 \mathrm{~mL})$ aliquots were obtained and the entire isolated small intestine as well as the mesenteric blood vessels were harvested for analyses of cyanidin-3-glucoside and its conjugates with RPHPLC with electrochemical (coulometric) detection after sample preparation as described below.

\section{Vascular samples}

Of each vascular sample, $1 \mathrm{~mL}$ was centrifuged at $10000 \mathrm{x} g$ for $10 \mathrm{~min}$ (Sigma 112, Sigma Laborzentrifugen $\mathrm{GmbH}$, Osterode, Germany). The supernatant was separated, the pellet resuspended with $0.1 \mathrm{~mL}$ ethanol and centrifuged again at $10000 \mathrm{xg}$ for $10 \mathrm{~min}$. The combined supernatants $(0.9 \mathrm{~mL})$ were analyzed by HPLC. Cyanidin-3-glucoside showed a recovery from vascular media of $101.4 \pm 3.7 \%$ (means $\pm S D, n=3$ ). After the perfusion experiment, cyanidin-3-glucoside was stabilized in the vascular samples by addition of $17.6 \mathrm{mg}$ ascorbic acid (resulting in a concentration of $2 \mathrm{mmol} / \mathrm{L}$ ) and $5 \mathrm{~mL}$ hydrochloric acid $(2 \mathrm{~mol} / \mathrm{L})$.

\section{Luminal samples}

After centrifugation at $2800 \mathrm{x} g$ for $20 \mathrm{~min}$ (Hermle ZK 364; Kontron, Zürich, Switzerland), the supernatant was separated. The pellet was extracted twice with acidified methanol/water (1:1, $200 \mathrm{mmol} / \mathrm{L}$ hydrochloric acid) by sonication for $10 \mathrm{~min}$ and then centrifuged again at $2800 \mathrm{x} g$ for $10 \mathrm{~min}$. The volume of the combined supernatants was defined and the solution was analyzed by HPLC. Cyanidin-3-glucoside recovery from luminal media was $90.2 \pm 0.4 \%$ (means $\pm \mathrm{SD}, \mathrm{n}=4$ ). After sampling the $\mathrm{pH}$ was immediately adjusted to 1 with $2 \mathrm{~mol} / \mathrm{L}$ hydrochloric acid.

\section{Small intestinal tissue and blood vessels}

After lyophilization of the entire small intestine, the tissue was cut into small pieces and subsequently extracted by sonication with $4.5 \mathrm{~mL}$ and $6.0 \mathrm{~mL}$ of an acidified methanol-water mixture $(1: 1,200 \mathrm{mmol} / \mathrm{L}$ hydrochloric acid). After centrifugation ( $10000 \mathrm{x} g$ for $10 \mathrm{~min}$ ), the extracts were pooled and the volume adjusted to $8-9 \mathrm{~mL}$ 
(the exact volume was defined). The blood vessels were lyophilized and extracted like the small intestinal tissue except that the extraction volume was $3.0 \mathrm{~mL}$ (twice) and the extracts were adjusted to about $5 \mathrm{~mL}$ (the exact volume was defined). Cyanidin-3-glucoside exhibited a recovery from the small intestinal tissue and blood vessels of $83.6 \pm 3.2 \%$ (means $\pm S D, n=3$ ).

\section{Analytical procedures}

\section{Gradient HPLC system with coulometric detection}

The HPLC system (Sykam, Gilching, Germany) consisted of a solvent delivery system S 1100, an HPLC controller S 2000, a low pressure gradient mixer S 8110, an autoinjector Marathon Basic $^{+}$(Spark, Emmen, The Netherlands) with a $100 \mu \mathrm{L}$ loop and an ESA Coulochem electrochemical detector (model 5100A with guard cell 5020 and analytical cell 5011; Max Stevenson scientific \& medical equipment, Berlin, Germany) at $+900 \mathrm{mV}$. Continuous on-line monitoring and data quantification was performed with a Chromatopac CR5A data processor (Shimadzu Corp., Kyoto, Japan). Separation was carried out on a Gromsil ODS-4 HE column $(125 \mathrm{~mm}$ x $4.0 \mathrm{~mm}$ I.D., $5 \mu \mathrm{m}$, Grom Analytik \& HPLC GmbH, Herrenberg, Germany), with a flow rate of $0.9 \mathrm{~mL} / \mathrm{min}$. Elution of cyanidin-3-glucoside (about $11 \mathrm{~min}$ ) and of aglycone cyanidin (about $19 \mathrm{~min}$ ) was achieved using the following gradient conditions: 0-3 min, 0\% B; 3-8 min, 0-12\% B; 12-17 min, $12-17 \%$ B; 17-24 min, $17-38 \% \mathrm{~B}$. Eluent A consisted of acetonitrile/water/formic acid $(3 / 87 / 10, \mathrm{v} / \mathrm{v} / \mathrm{v})$; eluent B was composed of acetonitrile/water/formic acid (50/40/10, $\mathrm{v} / \mathrm{v} / \mathrm{v})$. An injection volume of $50 \mu \mathrm{L}(10 \mu \mathrm{L}$ in case of luminal samples) resulted in a detection limit of $30 \mathrm{nmol} / \mathrm{L}$ and a quantification limit of $100 \mathrm{nmol} / \mathrm{L}$ for cyanidin-3glucoside. In case of the aglycone cyanidin, detection limit was $50 \mathrm{nmol} / \mathrm{L}$, quantification limit $150 \mathrm{nmol} / \mathrm{L}$.

Naringin (naringenin-7-glucoside) was used as an internal standard in concentrations of $2.46 \mathrm{mmol} / \mathrm{L}$ for luminal and $24.6 \mu \mathrm{mol} / \mathrm{L}$ for vascular samples as well as for small intestinal tissue and for blood vessels.

\section{Gradient HPLC system with MS detection (LC-MS)}

For the identification of cyanidin-3-glucoside and conjugates we used a clean-up procedure for vascular samples and gradient HPLC system combined with a MS detector in the electro-spray-ionization mode $\left(\mathrm{ESI}^{+}, \mathrm{ESI}^{-}\right)$ as described earlier [16]. Luminal samples were enriched tenfold by evaporation with nitrogen at $37^{\circ} \mathrm{C}$ prior to the LC-ESI-MS analysis.

\section{Cleavage of cyanidin-3-glucoside conjugates}

Conjugates such as glucuronides and sulfates were analyzed as cyanidin-3-glucoside after enzymatic cleavage according to Sfakianos et al. [17], with modifications as described below. For cleavage of glucuronides, $0.15 \mathrm{~mL}$ of $\mathrm{K}_{2} \mathrm{HPO}_{4}(1 \mathrm{~mol} / \mathrm{L}), 0.05 \mathrm{~mL} \mathrm{NaOH}(2 \mathrm{~mol} / \mathrm{L})$ and $0.1 \mathrm{~mL}$ glucuronidase solution (248 Fishman units; $E$. coli, Sigma-Aldrich, Deisenhofen, Germany) were added to $0.6 \mathrm{~mL}$ sample solution. Subsequent cleavage of sulfate conjugates was performed with $0.0625 \mathrm{~mL}$ glucuronidase-sulfatase solution (265 units; Helix pomatia, Sigma-Aldrich, Deisenhofen, Germany) and $0.5 \mathrm{~mL}$ sample solution (glucuronidase incubation) in an acetate buffer $(0.0625 \mathrm{~mL}, 0.2 \mathrm{~mol} / \mathrm{L}, \mathrm{pH} 4.5)$. Mixtures were incubated for $15 \mathrm{~min}$ (glucuronides) and $45 \mathrm{~min}$ (sulfates) at $37^{\circ} \mathrm{C}$. (Glucuronidase: $\beta$-D-glucuronide glucuronosohydrolase, EC 3.2.1.31; Sulfatase: aryl-sulfate sulfohydrolase, EC 3.1.6.1).

Since sulfate and glucuronide conjugates of anthocyanins are not commercially available, the applicability of the enzymatic cleavage in luminal perfusate was confirmed by the conversion of the sulfate and glucuronide conjugates of 4-nitrophenol. The cleavage of 4-nitrophenol glucuronide resulted in 4-nitrophenol recovery of $96.6 \pm 1.8 \%$ (mean $\pm S D, n=3$ ), the cleavage of 4-nitrophenol sulfate resulted in a recovery of $102.0 \pm 0.5 \%$ (mean $\pm S D, n=3$ ).

\section{Chemicals and solvents}

All chemicals used were of analytical grade. Solvents for HPLC analysis were of HPLC grade. Cyanidin-3-glucoside and cyanidin were purchased from Extrasynthèse (Genay, France), naringin, 4-nitrophenol, 4-nitrophenol-glucuronide and -sulfate from Fluka (Buchs, Switzerland).

\section{Calculations}

Oxygen and glucose consumptions were calculated from arterio-venous concentration differences $(\Delta C)$, the corresponding flow rates and the dry weight (DW) of the entire small intestine used in the experiment and given as fluxes $\left(\mu \mathrm{mol} \times \min ^{-1} \times(\mathrm{g} \text { dry intestine })^{-1}\right.$, means \pm $\mathrm{SD}$ ) according to the following equation:

$$
\text { Flux }=\frac{\Delta \mathrm{C}\left[\mathrm{nmol} \mathrm{mL}^{-1}\right] \times \text { flow }\left[\mathrm{mL} \mathrm{min}^{-1}\right]}{\mathrm{DW}[\mathrm{g}]}
$$

Statistical differences of absorption rates and viability parameters were determined using student's t-test for unpaired observations. $P$ values less than 0.05 were considered to indicate significant differences. 


\section{Results}

In control perfusion experiments with anthocyanin-free basic perfusion media, neither cyanidin-3-glucoside nor anthocyanin metabolites were detected. Stability of cyanidin-3-glucoside in the luminal $(95.5 \pm 6.8 \%, \mathrm{n}=3)$ and vascular perfusate $(91.6 \pm 13.6 \%, \mathrm{n}=3)$ was confirmed for $1.5 \mathrm{~h}$ at $37^{\circ} \mathrm{C}$. The recovery of cyanidin-3-glucoside from the perfusates and the tissues was nearly complete.

All organ preparations were viable during the perfusion experiments, confirmed by repeatedly measuring oxygen uptake, glucose-lactate handling and acid-base homeostasis. No significant differences were observed in comparing viability data from experiments with and without ethanol ${ }^{1}$.

In both experimental groups, most of the luminally

1 Viability parameters (means $\pm S D, n=5$ ): perfusion experiments without and with ethanol: oxygen consumption: $4.7 \pm 1.0$ (5.3 \pm 1.2) $\mu \mathrm{mol} \mathrm{x} \mathrm{min}{ }^{-1} \mathrm{x} \mathrm{g}^{-1}$, lactate-pyruvate ratio: $37.9 \pm 16.4(32.5 \pm$ 14.4); glucose consumption: $6.1 \pm 2.4(6.6 \pm 2.8) \mu \mathrm{mol} \mathrm{x} \mathrm{min}^{-1} \mathrm{x} \mathrm{g}^{-1}$; arterial pressure $67 \pm 6(68 \pm 11) \mathrm{mm} \mathrm{Hg}$; arterial pH: $7.51 \pm 0.03$ (7.48 \pm 0.02$)$; venous pH: $7.39 \pm 0.04(7.34 \pm 0.04)$. administered cyanidin-3-glucoside left the organ preparation via luminal efflux (Table 1). The main compound in the luminal and vascular effluent was unchanged cyanidin-3-glucoside. Small amounts of cyanidin-3-glucoside were absorbed in the conjugated form. Minor amounts of the applied substance and its conjugates were found in the gut tissue extracts. Enzymatic cleavage of perfusates and tissue extracts with glucuronidase yielded an increase in cyanidin-3-glucoside concentration. Sulfatase cleavage following glucuronidase incubation only yielded slightly higher cyanidin-3-glucoside concentrations in the intestinal tissue (Table 1). The enzymatic treatments indicate that the conjugates were predominantly glucuronides. However, LC-ESI-MS analyses enabled neither characterization of a sulfate nor a glucuronide-conjugate.

Absorption rates of cyanidin-3-glucoside with (4.3 \pm $3.2 \%)$ and without ethanol $(2.9 \pm 1.8 \%)$ were not significantly different. The recovery over five experiments with ethanol was $73.2 \%$ and $68.8 \%$ without ethanol, respectively.
Table 1 Distribution of cyanidin-3-glucoside and its conjugates in the luminal, vascular and tissue compartments after perfusion experiments of $1 \mathrm{~h}$ with the isolated rat small intestine ${ }^{1}$. N. D. is under detection limit

\begin{tabular}{|c|c|c|c|c|}
\hline & \multicolumn{2}{|l|}{ Without ethanol } & \multicolumn{2}{|l|}{ With ethanol } \\
\hline & $\mathrm{nmol} \pm \mathrm{SD}$ & $\% \pm S D^{2}$ & $\mathrm{nmol} \pm \mathrm{SD}$ & $\% \pm S D^{3}$ \\
\hline $\begin{array}{l}\text { Luminal loss } \\
\text { cyanidin-3-glucoside }\end{array}$ & $2197.7 \pm 402.7$ & $59.1 \pm 11.7$ & $2580.0 \pm 507.1$ & $66.8 \pm 6.1$ \\
\hline glucuronides & $251.9 \pm 363.9$ & $6.5 \pm 9.9$ & $27.0 \pm 60.3$ & $1.6 \pm 2.8$ \\
\hline sulfates & N. D. & & N. D. & \\
\hline total & $2449.6 \pm 515.6$ & $65.6 \pm 13.2$ & $2607.0 \pm 473.7$ & $68.4 \pm 9.0$ \\
\hline $\begin{array}{l}\text { Vascular uptake } \\
\text { cyanidin-3-glucoside }\end{array}$ & $100.9 \pm 64.4$ & $2.7 \pm 1.5$ & $118.4 \pm 66.3$ & $3.6 \pm 2.4$ \\
\hline glucuronides & $9.0 \pm 15.5$ & $0.2 \pm 0.4$ & $14.3 \pm 22.9$ & $0.7 \pm 0.9$ \\
\hline sulfates & N. D. & & N. D. & \\
\hline total & $109.9 \pm 79.1$ & $2.9 \pm 1.8$ & $132.7 \pm 84.0$ & $4.3 \pm 3.2$ \\
\hline $\begin{array}{l}\text { Intestinal tissue } \\
\text { cyanidin-3-glucoside }\end{array}$ & $11.1 \pm 6.1$ & $0.3 \pm 0.2$ & $9.8 \pm 8.7$ & $0.3 \pm 0.2$ \\
\hline glucuronides & N. D. & & $3.1 \pm 2.9$ & $0.1 \pm 0.1$ \\
\hline sulfates & N. D. & & $1.6 \pm 2.2$ & $0.1 \pm 0.1$ \\
\hline total & $11.1 \pm 6.1$ & $0.3 \pm 0.2$ & $14.5 \pm 11.0$ & $0.5 \pm 0.2$ \\
\hline $\begin{array}{l}\text { Blood vessels } \\
\text { cyanidin-3-glucoside }\end{array}$ & $1.2 \pm 0.8$ & $0.04 \pm 0.03$ & $1.3 \pm 1.2$ & $0.03 \pm 0.03$ \\
\hline glucuronides & $0.2 \pm 0.4$ & $0.004 \pm 0.01$ & $0.1 \pm 0.1$ & $0.002 \pm 0.004$ \\
\hline sulfates & N. D. & & N. D. & \\
\hline total & $1.4 \pm 1.0$ & $0.04 \pm 0.03$ & $1.4 \pm 1.1$ & $0.03 \pm 0.03$ \\
\hline recovery & $2572.0 \pm 527.4$ & $68.8 \pm 13.0$ & $2755.6 \pm 445.1$ & $73.2 \pm 8.2$ \\
\hline
\end{tabular}

$14294.0 \pm 1308.2 \mathrm{nmol}$ of cyanidin-3-glucoside with $5 \%$ ethanol and $3780.2 \pm 782.3 \mathrm{nmol}$ of cyanidin-3-glucoside without ethanol in luminal media were applied, each in five perfusion experiments

2 Based on the mean dosage of $3780.2 \pm 782.3 \mathrm{nmol}$

3 Based on the mean dosage of $4294.0 \pm 1308.2 \mathrm{nmol}$ 


\section{Discussion}

To assess the influence of ethanol on intestinal absorption and metabolism of cyanidin-3-glucoside, we used an isolated preparation of a vascularly and luminally perfused rat small intestine, characterized by fully maintained tissue viability. Intact mucosal morphology without loss of villous tip cells after 120 min perfusion has previously been demonstrated in histologic specimens of the isolated intestinal preparations perfused in the same way as in the present study [18]. As in earlier studies, viability and functional integrity of the intestinal preparation were carefully and continuously controlled $[14,15]$. The functional integrity of the intestinal preparation was monitored and the viability confirmed in agreement with data from earlier studies using the same model $[16,19,20]$.

Control perfusions with basic media without cyanidin-3-glucoside confirmed that the small intestine of the experimental animals did not contain any anthocyanins sequestered from food. It is also to be emphasized that in the present study the administered amount of cyanidin-3-glucoside (3780 and $4294 \mathrm{nmol}$ equivalent to 1.70 and $1.93 \mathrm{mg}$ ) was in the physiological range and might be easily taken up by a normal meal ( $30 \mathrm{mg}$ of elderberry [21], $2 \mathrm{~mL}$ of blood orange juice [22] or $5 \mathrm{~g}$ of sweet cherries [23].

The incomplete total recoveries of cyanidin-3-glucoside after perfusion experiments (about $70 \%$ ) was also observed in experimental studies with anthocyanins [24]. Cyanidin-3-glucoside might be metabolized to substances which escape electrochemical detection under the present conditions. In most anthocyanin studies no recoveries were indicated [6-11]. The incomplete recovery and moreover the low absorption rates might explain lack of quantitative data in the literature concerning absorption of anthocyanins.

The low absorption rate of cyanidin-3-glucoside and conjugates in the present study $(2.9 \%$ and $4.3 \%$, without and with ethanol, respectively) is in good agreement with earlier observations gained from human feeding experiments performed with mixtures of anthocyanins from red wine ranging from $1 \%$ to $6.7 \%$ [8]. In feeding experiments with rats, bioavailability of an anthocyanine mixture from Vaccinium myrtillus extracts was calculated somewhat lower (1.2\%) [6]. In contrast to these and to our results, absorption rate of pure cyanidin-3glucoside [9-11] and anthocyanins from elderberries [24] in human and animal studies was estimated to be extremely low.

From human studies, it was concluded that absorption of aglycones is enhanced by conjugation with glucose, possibly by absorption via the sodium-dependent glucose transport system (SGLT1) [25]. Absorption of anthocyanidin glycosides from black currant was suggested to occur through the hexose transport pathway
[10]. In contrast, the 7-glucoside of genistein, an isoflavone, was absorbed far less than the aglycone and a passive diffusion for the transepithelial transport was proposed $[19,20]$. For cyanidin-3-glucoside absorption, both transport processes are conceivable.

It is generally proposed that flavonoid glycosides are hydrolyzed to the corresponding less polar aglycones prior to gastrointestinal absorption [26-28] and an ability of mammals to hydrolyze flavonoid glycosides to the corresponding aglycones has been repeatedly reported [20,29-31]. However, cyanidin-3-glucoside was not hydrolyzed by small intestinal $\beta$-glucosidase and only traces (at the detection limit) of the aglycone cyanidin in a vascular perfusate were found. These results are in line with recent observations from human and animal studies $[9,11]$.

Conjugates of cyanidin-3-glucoside were analyzed after enzymatic cleavage. It must be emphasized that none of the enzymes, neither the glucuronidase from $E$. coli nor the glucuronidase-sulfatase from Helix pomatia, were able to cleave the $\beta$-glucoside cyanidin-3-glucoside, despite an apparent $\beta$-glucosidase activity of these enzymes [20]. Our observations were confirmed by incubation experiments with cyanidin-3-glucoside and $\beta$ glucosidase [11]. Irrespective of the presence of ethanol, enzymatic cleavage of perfusates and tissue extracts with glucuronidase yielded an increase in cyanidin-3glucoside concentration, which was not significantly different between the experimental groups. Subsequent incubation with glucuronidase-sulfatase did not further increase cyanidin-3-glucoside concentration. Therefore, we suppose formation of glucuronide or diglucuronide conjugates during perfusion experiments. With LC-ESImass spectrometry, however, we could not identify any such metabolites. Metabolites of cyanidin-3-glucosides have been also observed in an animal study [9], but so far no glucuronide conjugates have been characterized.

It has been supposed that the presence of ethanol in red wine might improve flavonoid availability [12]. It is conceivable that ethanol affects absorption by solubilizing anthocyanins or acting as a mucosal barrier breaker $[32,33]$. In the ethanol experiments, cyanidin-3-glucoside was solubilized in the luminal medium spiked with $5 \%$ ethanol. Wine contains about $12 \%$ ethanol, which is diluted during the digestion process. To investigate effects of ethanol on intestinal handling, we decided therefore, to work with a $5 \%$ ethanolic solution, as a convenient basis.

Our experiments clearly indicate that ethanol has no significant influence on absorption of the anthocyanin cyanidin-3-glucoside. For other phenolic compounds similar observations were reported. Human studies with dealcoholized and reconstituted red wine indicated that coingestion of ethanol did not affect extent of catechin [34, 35], caffeic, protocatechuic and 4-0-methyl gallic acid absorption [36]. 
Cyanidin-3-glucoside, this potent pharmacologically active plant component, is absorbed in the small intestine, mainly as intact $\beta$-glucoside. Yet, the extent of absorption is low. The present study under well-controlled experimental conditions reveals for the first time that ethanol has no influence on the amount of cyanidin-3glucoside absorbed. However, low absorption rates of anthocyanins obviously seem to be high enough to ex- ert beneficial physiological activity, reported from feeding studies [3-5].

Acknowledgments We are indebted to R.-P. Franke and W. Röhlke, Central Institute for Biomedical Technique, Department Biomaterials, University of Ulm, Germany, for the production of the perfluorocarbon emulsion and we thank W. Armbruster, Institute for Food Chemistry, University of Hohenheim, Stuttgart, Germany for performing the LC-MS analyses.

\section{References}

1. Paganga G, Miller N, Rice-Evans CA (1999) The polyphenolic content of fruit and vegetables and their antioxidant activities. What does a serving constitute? Free Radic Res 30:153-162

2. Ghiselli A, Nardini M, Baldi A, Saccini C (1998) Antioxidant activity of different phenolic fractions separated from an Italian red wine. J Agric Food Chem 46: 361-367

3. Joseph JA, Shukitt-Hale B, Denisova NA, Bielinski D, Martin A, McEwen JJ Bickford PC (1999) Reversals of age-related declines in neuronal signal transduction, cognitive, and motor behavioral deficits with blueberry, spinach, or strawberry dietary supplementation. J Neuroscience 19:8114-8121

4. Keevil JG, Osman HE, Reed JD, Folts JD (2000) Grape Juice, but not orange juice or grapefruit juice, inhibits human platelet aggregation. J Nutr 130:53-56

5. Lietti A, Forni G (1976) Studies on Vaccinium myrtillus anthocyanosides. Arzneim-Forsch (Drug Res) 26: 832-835

6. Morazzoni P, Livio S, Scilingo A, Malandrino S (1991) Vaccinium myrtillus anthocyanosides pharmacokinetics in rats. Arzneim-Forsch (Drug Res) 41: 128-131

7. Cao G, Prior RL (1999) Anthocyanins are detected in human plasma after oral administration of an elderberry extract. Clin Chem 45:574-576

8. Lapidot T, Harel S, Granit R, Kanner J (1998) Bioavailability of red wine anthocyanins as detected in human urine. J Agric Food Chem 46:4297-4302

9. Tsuda T, Horio F, Osawa T (1999) Absorption and metabolism of cyanidin 3 O-beta-D-glucoside in rats. FEBS Lett 449:179-182

10. Matsumoto H, Inaba H, Kishi M, Tominaga S, Hirayama $M$, Tsuda T (2001) Orally administered delphinidin 3-rutinoside and cyanidin 3-rutinoside are direct absorbed in rats and humans and appear in the blood as the intact forms. J Agric Food Chem 49:1546-1551
11. Miyazawa T, Nakagawa K, Kudo M, Muraishi K, Someya K (1999) Direct intestinal absorption of red fruit anthocyanins, cyanidin-3-glucoside and cyanidin-3,5-diglucoside, into rats and humans. J Agric Food Chem 47: 1083-1091

12. Ruf J-C, Berger J-L, Renaud S (1995) Platelet rebound effect of alcohol withdrawal and wine drinking in rats. Arterioscler Thromb Vasc Biol 15:140-144

13. Hummel M, Pogan $K$, Stehle $P$, Fürst $P$ (1997) Intestinal taurine availability from synthetic amino acid-taurine conjugates: an in vitro perfusion study in rats. Clin Nutr 16:137-139

14. Plauth M, Raible A, Bauder-Gross D, Vieillard-Baron D, Fürst P, Hartmann F (1991) Effects of dexamethasone on glutamine metabolism in the isolated vascularly perfused rat small intestine. Res Exp Med 191:349-357

15. Hartmann F, Vieillard-Baron D, Heinrich R (1984) Isolated perfusion of the small intestine using perfluorotributylamine as artificial oxygen carrier. Adv Exp Med Biol 180:711-720

16. Andlauer W, Kolb J, Siebert K, Fürst P (2000) Assessment of resveratrol bioavailability in the perfused small intestine of the rat. Drugs Exptl Clin Res 26:47-55

17. Sfakianos J, Coward L, Kirk M, Barnes S (1997) Intestinal uptake and biliary excretion of the isoflavone genistein in rats. J Nutr 127:1260-1268

18. Plauth M, Kremer I, Raible A, Stehle P, Fürst P, Hartmann F (1992) Nitrogen absorption from isonitrogenous solutions of L-leucyl-L-leucine and Lleucine: a study in the isolated perfused rat small intestine. Clin Sci 82:283-290

19. Andlauer W, Kolb J, Stehle P, Fürst P (2000) Absorption and metabolism of genistein in the isolated rat small intestine. J Nutr 130:843-846

20. Andlauer W, Kolb J, Fürst P (2000) Absorption and metabolism of genistin in the isolated rat small intestine. FEBS Lett 465:127-130
21. Abuja PM, Murkovic M, Pfannhauser W (1998) Antioxidant and prooxidant activities of elderberry (Sambucus nigra) extract in low density lipoprotein oxidation. J Agric Food Chem 46: 4091-4096

22. Bridle P, Timberlake CF (1997) Anthocyanins as natural food colours - selected aspects. Food Chem 58:103-109

23. Gao L, Mazza G (1995) Characterization, quantitation, and distribution of anthocyanins and colorless phenolics in sweet cherries. J Agric Food Chem 43:343-346

24. Murkovic M, Adam U, Pfannhauser W (2000) Analysis of anthocyane glycosides in human serum. Fresenius J Anal Chem 366:379-381

25. Hollman PC, Bijsman MN, vanGameren Y, Cnossen EP, deVries JH, Katan MB (1999) The sugar moiety is a major determinant of the absorption of dietary flavonoid glycosides in man. Free Radic Res 31:569-573

26. Manach C, Regerat F, Texier O, Agullo G, Demigne C, Remesy C (1996) Bioavailability, metabolism and physiological impact of 4-oxo-flavonoids. Nutr Res 16:517-544

27. Yasuda T, Mizunuma S, Kano Y, Saito K, Oshawa K (1996) Urinary and biliary metabolites of genistein in rats. Biol Pharm Bull 19:413-417

28. Setchell KD, Zimmer-Nechemias L, Cai J, Heubi JE (1998) Isoflavone content of infant formulas and the metabolic fate of these phytoestrogens in early life. Am J Clin Nutr 68:1453S-1461S

29. Booth AN, Jones FT, DeEds F (1957) Metabolic fate of hesperidin, eriodictyol, homoeriodictyol, and diosmin. J Biol Chem 16:661-668

30. Day AJ, DuPont MS, Ridley S, Rhodes M, Rhodes MJ, Morgan MR, Williamson G (1998) Deglycosylation of flavonoid and isoflavonoid glycosides by human small intestine and liver beta-glucosidase activity. FEBS Lett 436:71-75 
31. Ioku K, Pongpiriyadacha Y, Konishi Y, Takei Y, Nakatani N, Terao J (1998) betaGlucosidase activity in the rat small intestine toward quercetin monoglucosides. Biosci Biotechnol Biochem 62: 1428-1431

32. Beck IT, Morris GP, Buell MG (1986) Ethanol-induced vascular permeability changes in the jejunal mucosa of the dog. Gastroenterology 90:1137-1145
33. Morris GP, Beck PL, Herridge MS, Depew WT, Szewczuk MR, Wallace JL (1989) Hapten-induced model of chronic inflammation and ulceration in the rat colon. Gastroenterology 96: 795-803

34. Bell JR, Donovan JL, Wong R, Waterhouse AL, German JB, Walzem RL, Kasim-Karakas SE (2000) (+)-Catechin in human plasma after ingestion of a single serving of reconstituted red wine. Am J Clin Nutr 71:103-108
35. Donovan JL, Bell JR, Kasim-Karakas S, German JB, Walzem RL, Hansen RJ, Waterhouse AL (1999) Catechin is present as metabolites in human plasma after consumption of red wine. J Nutr 129: 1662-1668

36. Caccetta RA, Croft KD, Beilin LJ, Puddey IB (2000) Ingestion of red wine significantly increases plasma phenolic acid concentrations but does not acutely affect ex vivo lipoprotein oxidizability. Am J Clin Nutr 71:67-74 